\title{
Effect of prenatal different auditory environment on learning ability and fearfulness in chicks
}

\author{
Shuai Zhao ${ }^{1}$, Chunzhu $\mathrm{Xu}^{2}$, Runxiang Zhang ${ }^{1,3}$, Xiang $\mathrm{Li}^{1}$, Jianhong $\mathrm{Li}^{2}{ }^{2, *}$, and Jun Bao ${ }^{1,3, *}$
}

\footnotetext{
* Corresponding Authors:

Jianhong Li

Tel: +86-451-55190114,

Fax: +86-451-55190114,

E-mail: jhlineau@126.com

Jun Bao

Tel: +86-451-55190114,

Fax: +86-451-55190114

E-mail: jbao@neau.edu.cn
}

${ }^{1}$ College of Animal Science and Technology, Northeast Agricultural University, Harbin 150030, Heilongjiang, China

${ }^{2}$ College of life Science, Northeast Agricultura

University, Harbin 150030, Heilongjiang,

China

${ }^{3}$ Key Laboratory of Chicken Genetics and Breeding, Ministry of Agriculture and Rural Affairs, Harbin 150030, China

ORCID

Shuai Zhao

https://orcid.org/0000-0003-1518-647X

Chunzhu Xu

https://orcid.org/0000-0002-9993-7666

Runxiang Zhang

https://orcid.org/0000-0002-6315-9399 Xiang Li

https://orcid.org/0000-0001-8944-086X

Jianhong Li

https://orcid.org/0000-0003-1366-1089

Jun Bao

https://orcid.org/0000-0002-9492-0507

Submitted Oct 14, 2021; Revised Nov 12, 2021; Accepted Dec 18, 2021
Objective: Early environmental enrichment in life can improve cognition in animals. The effect of prenatal auditory stimulation on learning ability and fear level in chick embryos remained unexplored. Therefore, this study investigated the effect of prenatal auditory stimulation on the learning ability and fear level of chicks.

Methods: A total of 450 fertilized eggs were randomly divided into 5 groups, including control group (C), low-sound intensity music group (LM), low-sound intensity noise group (LN), high-sound intensity noise group (HN) and high-sound intensity music group (HM). From the 10th day of embryonic development until hatching, group LM and group LN received 65 to $75 \mathrm{~dB}$ of music and noise stimulation. Group HN and group HM received 85 to $95 \mathrm{~dB}$ of noise and music stimulation, and group $\mathrm{C}$ received no additional sound. At the end of incubation, the one-trial passive avoidance learning (PAL) task and tonic immobility (TI) tests were carried out, and the serum corticosterone (CORT) and serotonin (5-HT) concentrations were determined.

Results: The results showed that compared with the group C, 65 to $75 \mathrm{~dB}$ of music and noise stimulation did not affect the PAL avoidance rate $(p>0.05)$, duration of TI $(p>0.05)$ and the concentration of CORT $(\mathrm{p}>0.05)$ and 5-HT $(\mathrm{p}>0.05)$ in chicks. However, 85 to $95 \mathrm{~dB}$ of music and noise stimulation could reduce duration of TI $(\mathrm{p}<0.05)$ and the concentration of CORT $(\mathrm{p}<0.05)$, but no significant effect was observed on the concentration of 5-HT ( $p>0.05)$ and PAL avoidance rate $(\mathrm{p}>0.05)$.

Conclusion: Therefore, the prenatal auditory stimulation of 85 to $95 \mathrm{~dB}$ can effectively reduce the fear level of chicks while it does not affect the learning ability.

Keywords: Auditory Stimulation; Chicks; Fearfulness; Learning Ability

\section{INTRODUCTION}

Environmental enrichment may enhance the brain's normal development and the plasticity of brain structure and function and improve cognitive ability [1]. High cognitive ability can help animals to adapt to the changed social environment. Environmental enrichment is known to reduce the fearfulness of animals. For instance, chicks provide various novel stimuli, such as balls, buttons, thimbles, which can reduce fear in chicks. Fearfulness is an evolutionarily adaptive ability against danger [2]. Exaggerated fear responses may cause smothering and mortality in animals, which is a general concern in the poultry industry.

Prenatal environmental enrichment affects brain development and behavioral expression in animals [3]. For example, light [4] and sound [5] showed important prenatal experience that affects chick's behavior. In particular, prenatal auditory stimulation can result in the early maturation of the auditory system [6] and improve the learning ability of newborn animals [7]. Studies have shown that prenatal exposure to the sound results in increased neurogenesis in the hippocampus and enhance the spatial learning ability of rats [8] and 
chicks [3]. Thus, prenatal auditory stimulation plays a vital role in the development of neonates.

In auditory stimuli, music has a high functional value. Music exposure was considered an effective way to enrich the auditory system. As a result, music has been increasingly used as a form of acoustic stimulation to improve fowls' neurological development and welfare during incubation. For example, $65 \mathrm{~dB}$ and $110 \mathrm{~dB}$ of music exposure in the prenatal period can improve spatial learning ability by increasing the expression of hippocampal synaptic protein [6] and brain-derived neurotrophic factor (BDNF) [5]. In artificial incubation, there are many technologies, such as ventilation, egg flipping, humidity and refrigeration systems used to ensure optimal conditions for the development of poultry embryos. During this process, noise caused by the engine and fan was inevitable, which results in a sound intensity approach up to $95 \mathrm{~dB}$ [9]. During the incubation period, $110 \mathrm{~dB}$ noise exposure can affect the development of the poultry brain, reduce brain volume and weight [10], increase thyroxine, and impair learning and memory ability [11]. In other studies, the noise of commercial hatcheries at $90 \mathrm{~dB}$ was beneficial to artificial hatching, led to earlier hatching, higher hatchability, better chick quality [12]. Furthermore, there is evidence that the species-specific calls can also accelerate the hatching process and improving the hatching quality, which was beneficial to the anti-stress response and spatial orientation ability of day-old chicks $[12,13]$.

There are several studies on the effects of sound exposure on neurodevelopmental and incubation responses to the animals. A few studies were focused on the effect of prenatal sound exposure on fearfulness and the learning ability of newborn animals. Being a precocial species, chicks will exhibit responses to air borne sound around embryonic days 11/12 [14]. Meanwhile, they have strong learning ability and memory on the first day after incubation, enabling them to complete various cognitive tasks and show a preference for sound [15]. Thus, we choose chicks as the experimental model. The aim of this study determines whether the addition of auditory stimuli in the prenatal of poultries could reduce fear and improve cognitive ability by detection of the onetrial passive avoidance learning (PAL) task, tonic immobility (TI) test and the concentrations of serotonin and corticosterone in neonatal chicks.

\section{MATERIALS AND METHODS}

\section{Subjects and general procedures}

All experiments were approved by and conducted according to the guidelines of the Institutional Animal Care and Use Committee of Northeast Agriculture University (Ethic code: IACUCNEAU20190717).

Roman-white domestic chicks (Gallus gallus domesticus) were used as the experimental model. Fertilized eggs (day 0) of healthy laying hens (weigh of $60 \pm 5 \mathrm{~g}$ ) were obtained from a local registered commercial rearing unit and incubated in an incubator (Incubator-LN2-S, Dezhou Limin Livestock Machinery Co., Ltd., Shandong, China). Incubation conditions were maintained at $50 \%$ to $70 \%$ humidity and temperature of $37^{\circ} \mathrm{C}\left( \pm 1^{\circ} \mathrm{C}\right)$. The levels of temperature and humidity, and air quality in the incubator were controlled. The lighting schedule was $12 \mathrm{~L}: 12 \mathrm{D}$, and eggs automatically turned on every $2 \mathrm{~h}$ throughout the incubation period $(21 \mathrm{~d})$, which was done to prevent adhesions of embryos. The development of the embryo can be exposed to the egg lamp on the 8 th day, in which we examined all eggs and removed the unfertilized eggs.

\section{Experimental groups and auditory stimulation}

A total of 450 fertilized eggs were divided into the following five groups ( $\mathrm{n}=90$ for each group) and placed in five separate incubations:

Group C: Control group; the eggs were incubated under normal conditions with no additional sound exposure.

Group LM: Low-intensity music group; the eggs were exposed to Mozart's classical music (Mozart's String Quartets, K428, K525, K458) stimulus of intensity ranged from 65 to $75 \mathrm{~dB}$ from 10th day until hatching.

Group LN: Low-intensity noise group; the eggs were exposed to the noise stimulus of intensity ranged from 65 to $75 \mathrm{~dB}$ from 10th day until hatching. The noise was the prerecorded sound of ventilation fans and the commercial incubator.

Group HN: High-intensity noise group; the eggs were exposed to the noise stimulus of intensity ranged from 85 to $95 \mathrm{~dB}$ from 10th day until hatching. The noise was the pre-recorded sound of ventilation fans and the commercial incubator.

Group HM: High-intensity music group; the eggs were exposed to Mozart's classical music (Mozart's String Quartets, $\mathrm{K} 428, \mathrm{~K} 525, \mathrm{~K} 458$ ) stimulus of intensity ranged from 85 to $95 \mathrm{~dB}$ from 10th day until hatching.

During this period, experimental groups were exposed to sounds every day from 7:00 to 19:00. During this period, the sounds were played (on for an hour/off for an hour) in loops through two built-in speakers (SPA311; Philips (China) Investment Co., Ltd., Shanghai, China) connected to a laptop computer provided with an automatic setting. The intensity of the sounds emitted by the speakers was calibrated from measurements taken by a decibel meter (VICTORVC824; Shenzhen city station win Technology Co. Ltd., Guangdong, China) at the center of the incubation chamber ( $24 \mathrm{cms}$ apart).

All incubators produced about $45 \mathrm{~dB}$ sound intensity. 
However, the sound intensity was so far below than that of the experimental group. So, the possibility of acoustic interference was excluded.

\section{The one-trial passive avoidance learning task}

The one-trial PAL task exploits the spontaneous tendency of chicks to peck at objects for examining learning and memory in the young chicks [16].

After hatching, the chicks of five groups were maintained in five cartons $(38 \times 31 \times 25 \mathrm{~cm})$ and located in a temperaturecontrolled room $\left(32^{\circ} \mathrm{C}\right.$ to $\left.35^{\circ} \mathrm{C}\right)$. Ad libitum water was given, but the chicks were fasted. On the second day, 24 chicks (male: female $=1: 1$ ) from each treatment group were randomly selected and transferred to cartons $(20 \times 20 \times 25 \mathrm{~cm})$ in randomly assigned pairs, and each chick was illuminated with a $25 \mathrm{~W}$ LED light. Before pre-training, they were familiarized with the experimental environment at $30^{\circ} \mathrm{C}$ to $32^{\circ} \mathrm{C}$ for at least 30 min. In each pair, one chick was marked by spraying black color on its head for identification during the data recording.

At pre-training, the chicks were presented with a waterdipped white bead ( $2 \mathrm{~mm}$ diameter) for $10 \mathrm{~s}$ to encourage the chicks' natural pecking response. There was 5 min difference between each trial in this procedure, and three pretraining trials were performed. Each chick was presented with a red bead (4 mm diameter) dipped with $99 \%$ methyl anthranilate (MeA) for $10 \mathrm{~s}$ after $25 \mathrm{~min}$ of pre-training trial. MeA is a substance of aversive taste, and chickens typically respond to it with a disgust response, such as shaking their heads, closing their eyes, and occasionally wiping their beaks on the floor of the box. Test of memory was conducted at 120 min after training. The chicks were presented first with a dry red bead (identical to the one in the training trial), and then a dry white bead (identical to the one in the pretraining trial) on two trials $(10 \mathrm{~s}), 5 \mathrm{~min}$ interval. In order to exclude the influence of order and color, the first 6 pairs of chicks were presented with red beads, followed by white beads, and the last 6 pairs were presented with white beads and then red beads. Each chick's behavior was recorded as pecking or not pecking.

The memory retention was calculated as a percent avoidance score (the number of chicks in each group that avoids the red bead but pecks the white on test $\times 100 \% /$ total number of the trained chicks) [17]. Each chick was trained and tested only once. During the PAL task, the chicks which did not peck during at least one of the three pre-training trials, were excluded from the analysis in the training trial or the second testing trials.

\section{Tonic immobility}

On the second day of hatching, 20 chicks (male:female $=1: 1$ ) were randomly collected from each group and were tested individually for the duration of TI in a separate room. The chicks which had previously been tested in the PAL task and identified due to the black spray paint on its head, were not selected for the TI test. The TI test was carried out according to the method described previously [18]. Briefly, the individual chick was placed on its back in a wooden, $U$-shaped cradle. The chick was then restrained with one hand on its sternum while holding the head and neck with the other hand to induce TI. The induction time was $15 \mathrm{~s}$. The experimenter slowly released his hands, and the duration of TI reaction, that was, the freeze, was recorded. Freezing was defined as the absence of movement, except for any movement related to respiration and tremors. At the end of the induction period, hand pressure was gradually lifted so that if the chick could still move. Another induction period was started immediately, until the movement ceased. After removal of the hands, a stopwatch was started. The experimenter was then retreated, and the behavior of the chick was observed. If the chick righted itself in less than $10 \mathrm{~s}$, it was considered that TI had not been induced, and the restraint procedure was repeated (maximum three times). If a chick did not show a righting response over the $15 \mathrm{~min}$ test period, a maximum score of $900 \mathrm{~s}$ was given for duration.

\section{Sample collection and estimation of blood \\ corticosterone and serotonin concentration}

Tissue samples were collected after the TI and PAL tasks on the second day of hatching. Eight chicks (male:female $=1: 1$ ) were randomly selected from each group and decapitated immediately for tissue collection. The time between catching and tissue sampling was less than 2 min per chick. Blood was collected into $1.5 \mathrm{~mL}$ Eppendorf (EP) tubes. The blood was clotted for $24 \mathrm{~h}$ at $4^{\circ} \mathrm{C}$, and the EP tubes were spun down in an ALLSHENG iCen24 centrifuge (Hangzhou Allsheng Instruments Co., Ltd., Hangzhou, China) for $15 \mathrm{~min}$ at 4,000 rpm to separate the clot. The serum samples were poured off into $1.5 \mathrm{~mL} \mathrm{EP}$ tubes and stored at $-80^{\circ} \mathrm{C}$ until further analysis. The concentration of serum corticosterone (CORT) and serotonin (5-HT) was measured using a commercially available ELISA kit (Nanjing Jiancheng Bioengineering Institute, Nanjing, China) and the corresponding procedures. The intraassay coefficient of variation of the kit was less than $12 \%$, and the assay ranged from 10.2 to $60 \mathrm{ng} / \mathrm{mL}$.

\section{Statistical analysis}

Statistical analyses were performed using SPSS 21 for Windows (SPSS Inc., Chicago, IL, USA). All data were tested for normal distribution using the Kolmogorov-Smirnov test. Intergroup differences at the TI duration, CORT and 5-HT concentrations, were analyzed by unpaired Student's t-test. Fisher's Exact Test was conducted on memory retention. The results were expressed as mean \pm standard error of the mean, and differences were considered statistically significant at 
$\mathrm{p}<0.05$.

\section{RESULTS}

Effect of prenatal sound exposure for PAL task in chicks

The avoidance ratio for chicks from the control (C), low-intensity music (LM), low-intensity noise (LN), high-intensity noise (HN) and high-intensity music (HM) groups were presented in (Table 1). There were no significant differences in avoidance ratio among the groups (LM, p = 1), (LN, p = 1), ( $\mathrm{HN}, \mathrm{p}=0.422)$, and $(\mathrm{HM}, \mathrm{p}=0.214)$ compared to group C. In total, $22.5 \%$ of the chicks did not meet the inclusion criteria and were excluded from the analysis.

\section{Effect of prenatal sound exposure for tonic immobility}

Table 1. The effects of prenatal auditory exposure on learning in dayold chicks

\begin{tabular}{lccc}
\hline Treatment $^{1)}$ & $\mathbf{N}$ & Avoidance (\%) & p-value \\
\hline C & 16 & 68.75 & - \\
LM & 20 & 65 & 1 \\
LN & 19 & 68.42 & 1 \\
HN & 20 & 85 & 0.422 \\
HM & 18 & 88.89 & 0.214 \\
\hline
\end{tabular}

Results presented as percent avoidance for the passive avoidance ( $=$ the number of chicks in each group that avoided the red bead but pecked the white on test $\times 100 \%$ /total number of the trained chicks) learning tasks at 120 min after training. Numbers of chicks in each group are presented in the $\mathrm{N}$.

Statistical difference is represented as $p$ by Fisher's Exact Test compared to group $\mathrm{C}$.

${ }^{1)} \mathrm{C}$, control group; LM, low-sound intensity music group; LN, low-sound intensity noise group; HN, high-sound intensity noise group; HM, highsound intensity music group. in chicks

Mean values for the duration of tonic immobility per treatment were presented in (Figure 1). The group HM $(\mathrm{p}<0.05)$ and group $\mathrm{HN}(\mathrm{p}<0.05)$ displayed significantly shorter duration of tonic immobility compared to chicks from group C. There was no significant difference in TI duration in group LM ( $p>0.05)$ and group LN ( $>0.05)$ compared to group C.

\section{Effect of prenatal sound exposure for levels of serum} corticosterone and serotonin in chicks

The CORT concentration $(\mathrm{ng} / \mathrm{mL}$, mean \pm standard deviation $[\mathrm{SD}]$ ) of chicks in $\mathrm{C}, \mathrm{LM}, \mathrm{LN}, \mathrm{HN}$, and HM groups was presented in (Figure 2). This study showed that the chicks in group HN $(\mathrm{p}<0.05)$ and group HM $(\mathrm{p}<0.05)$ had significantly lower serum corticosterone concentrations than group

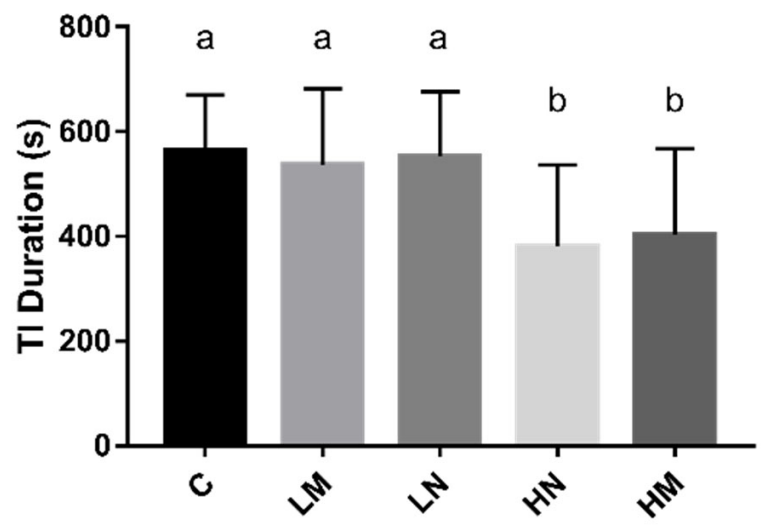

Figure 1. The effect of prenatal sounds exposure on TI duration (s) in chicks. $n=20 /$ treatment. TI, tonic immobility; ANOVA, analysis of variance. $^{\mathrm{a}, \mathrm{b}}$ Means with different superscripts represents significant differences between groups $(p<0.05)$, the same superscripts represent no significant differences $(p>0.05)$.

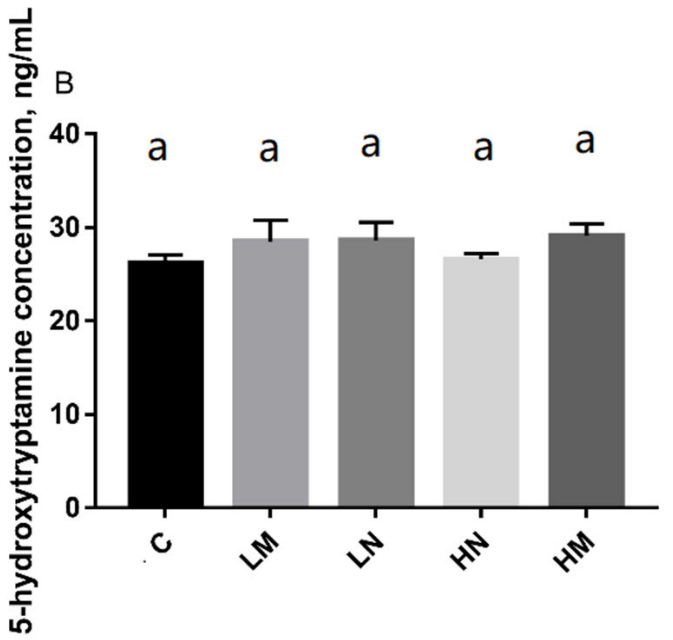

Figure 2. The effect of prenatal auditory exposure on CORT and $5-H T$ concentration $(n g / m L)$ in chicks. $n=8 /$ treatment. ANOVA, analysis of variance. ${ }^{a, b}$ Means with different superscripts represents significant differences between groups $(p<0.05)$, the same superscripts represent no significant differences ( $p>0.05)$. CORT, serum corticosterone; $5-H T$, serotonin. 
$\mathrm{C}$, but there were no significant differences among the LM ( $p>0.05), \mathrm{LN}(\mathrm{p}>0.05)$ and $\mathrm{C}$ groups.

The serum serotonin (5-HT) concentration $(\mathrm{ng} / \mathrm{mL}$, mean \pm SD) of chicks in the C, LM, LN, HN, and HM groups was presented. There were no significant differences in 5-HT concentration among the groups LM ( $>0.05), \mathrm{LN}(\mathrm{p}>0.05)$, $\mathrm{HN}(\mathrm{p}>0.05)$, and HM ( $>0.05)$ compared to group C.

\section{DISCUSSION}

The majority of the chicken auditory system development occurs in ovo and matures earlier than other sensory systems [19]. Therefore, sound exposure of prenatal, as an environmental enrichment method, provides an environmental stimulus for developing the avian sensory system. In this study, there were no differences in the PAL avoidance rate, duration of TI, CORT, and 5-HT concentrations of chicks between group C, group LM, and group LN. However, those of group HM and group HN were significantly difference than groups $\mathrm{C}, \mathrm{LN}$, and LM, which may be due to the acoustic insulation effect of eggshells on sound. Intact eggshells presented an insulation value of $17.14 \%$ at 70 $\mathrm{dB}$ that measured at the egg chamber with a special microphone [9]. This results in sound intensity after passing through the eggshell would not be enough to elicit a response from the embryo at 65 to $75 \mathrm{~dB}$. Tong et al [20] found that exposure to $72 \mathrm{~dB}$ did not affect the growth, hatchability, mortality and hormone level of the chicken embryos, which was also consistent with our results. Therefore, no significant effect was observed on chicken embryos exposure to 65 to $75 \mathrm{~dB}$ music and noise.

Environmental factors can influence the development of the brain throughout embryonic developmental stage in humans and other animals. Prenatal music stimulation promotes the expression of hippocampal synaptogenesis proteins and BDNF, enhancing spatial orientation [7] and cognitive abilities [21] of chicks, even when the sound intensity of music reaches $110 \mathrm{~dB}$ [6]. In this study, exposed to 85 to $95 \mathrm{~dB}$ music, the chicks did not show a higher avoidance rate in the PAL task. This may be because the sound intensity was not strong enough to elicit a response from the embryo in learning ability and memory. Interestingly, in this study found that prenatal exposure to 85 to $95 \mathrm{~dB}$ noise made the same effect in the PAL task.

Fear response of poultry has been increasingly used to evaluate the welfare status of poultry [22]. The duration of TI is a well-validated method to evaluate the fear level of poultry [23]. The longer the duration of immobility of poultry, the stronger the fear [22]. Environmental enrichment effectively decreases stress and fearfulness and improves the physical and psychological well-being of hens [24]. Sound (rhythmic and patterned) stimulus as an environmental en- richment method during hatching, it can reduce the fear level of chicks after hatching $[6,19]$. In this study, there were a significantly shorter TI duration of prenatal exposure to 85 to $95 \mathrm{~dB}$ music and noise environment was observed compared with the control group in day-old chicks, which suggests that the prenatal exposure to 85 to $95 \mathrm{~dB}$ music and noise could reduce the fear level.

In this study, there was no significant difference in the concentration of 5-HT between the treatment groups. However, the serum CORT concentration of chicks under prenatal exposure of 85 to $95 \mathrm{~dB}$ music and noise was significantly lower than that in the control group. CORT and 5-HT are the indicators of various forms of stress in fowls [25]. The concentrations of CORT in the blood were associated with thirst, hunger, heat, fear, stress or the barren environment in chicks [26]. The lower CORT concentration indicates a lower stress level. This is consistent with the decrease of CORT concentration caused by 85 to $95 \mathrm{~dB}$ music and noise stimuli in this study. Fear has been shown to cause changes in 5-HT concentrations [27]. However, in this study, the distinction of the degree of fear was not reflected accurately in the concentration of 5-HT. This may be because a large amount of 5 -HT in the blood is mainly carried by platelets [28], and serum 5-HT concentration is not the best choice. In future studies, whole blood 5-HT concentration should be selected to provide a basis for physiological measurement [29].

In summary, whether the effects of auditory stimulation during egg incubation are beneficial or detrimental well depend on rhythmicity and timing of exposure [12,14,27,28]. In this study, the music was the complex rhythmic sound, and the noise was the mixed noise of fans and machinery in the incubator, which was patterned sound. At a higher intensity, these patterned and rhythmic sounds have no harmful effects on the embryonic development of the auditory system, and spatial orientation and the ability to resist stress $[3,28$ 30]. However, in other studies, unpredictable noise, such as vehicle honking, was used [6,30-32]. These dissonant and noisy structures are associated with aggression, fear and defense $[27,28]$, which may be the main reason for the different results. In addition, the study of Hedlund et al [33] found that chicks have elevated levels of CORT hatched in commercial hatchery (the noise was ventilation fans at $90 \mathrm{~dB}$ ) compared to chicks hatched under calm conditions. It is contrary to our results, which may be because the commercial hatchery noise caused by fans was continuous for $24 \mathrm{~h} / \mathrm{d}$ during the incubation period, while the sound stimulation used in this study is intermittent for $6 \mathrm{~h} / \mathrm{d}$, which was far less than the time of exposure in commercial hatchery. This also shows that long-term continuous sound exposure before hatching is harmful to the development of embryonic, while intermittent short-term sound stimulation can have a beneficial effect. Further research should explore the effects of 
different timing of sound exposure in incubation period for newborn chicks.

\section{CONCLUSION}

This study indicated that prenatal exposure to 65 to $75 \mathrm{~dB}$ music and patterned noise could not produce effective stimulation for embryonic development. However, beneficial effects on embryonic development for chicks prenatally exposed to music and patterned noise were observed at 85 to $95 \mathrm{~dB}$ ( $6 \mathrm{~h}$ per day, on for an hour/off for an hour), reducing the fear level and serum CORT concentration, but there was no effect on the learning ability. Our study suggests that prenatal 85 to $95 \mathrm{~dB}$ music and patterned noise stimulation can positively reduce fearfulness in chicks.

\section{CONFLICT OF INTEREST}

We certify that there is no conflict of interest with any financial organization regarding the material discussed in the manuscript.

\section{FUNDING}

This work was supported by the National Natural Science Foundation of China (31772645).

\section{REFERENCES}

1. Nozari M, Shabani M, Hadadi M, Atapour N. Enriched environment prevents cognitive and motor deficits associated with postnatal MK-801 treatment. Psychopharmacology (Berl) 2014;231:4361-70. https://doi.org/10.1007/s00213014-3580-8

2. Jones RB, Waddington D. Modification of fear in domestic chicks, Gallus gallus domesticus, via regular handling and early environmental enrichment. Anim Behav 1992;43: 1021-33. https://doi.org/10.1016/S0003-3472(06)80015-1

3. Kauser H, Roy S, Pal A, Sreenivas V, Mathur R, Wadhwa S, Jain S. Prenatal complex rhythmic music sound stimulation facilitates postnatal spatial learning but transiently impairs memory in the domestic chick. Dev Neurosci 2011;33:4856. https://doi.org/10.1159/000322449

4. Belnap SC, Lickliter R. Prenatal light exposure influences gait performance and body composition in bobwhite quail chicks. Physiol Behav 2019;212:112706. https://doi.org/10. 1016/j.physbeh.2019.112706

5. Chaudhury S, Wadhwa S. Prenatal auditory stimulation alters the levels of CREB mRNA, p-CREB and BDNF expression in chick hippocampus. Int J Dev Neurosci 2009;27:583-90. https://doi.org/10.1016/j.ijdevneu.2009.06.004

6. Sanyal T, Kumar V, Nag TC, Jain S, Sreenivas V, Wadhwa S.
Prenatal loud music and noise: differential impact on physiological arousal, hippocampal synaptogenesis and spatial behavior in one day-old chicks. PLoS One 2013;8:e67347. https://doi.org/10.1371/journal.pone.0067347

7. Chaudhury S, Jain S, Wadhwa S. Expression of synaptic proteins in the hippocampus and spatial learning in chicks following prenatal auditory stimulation. Dev Neurosci 2010; 32:114-24. https://doi.org/10.1159/000279758

8. Faatehi M, Basiri M, Nezhadi A, et al. Early enriched environment prevents cognitive impairment in an animal model of schizophrenia induced by MK-801: Role of hippocampal BDNF. Brain Res 2019;1711:115-9. https://doi.org/10.1016/ j.brainres.2019.01.023

9. Donofre AC, Silva IJO, DeCastro Júnior SL. The sensor to estimate the sound pressure level in eggs. Comput Electron Agric 2018;154:420-5. https://doi.org/10.1016/j.compag.2018. 09.027

10. Alpana GK. Effect of prenatal chronic noise exposure on the growth and development of body and brain of chick embryo. Int J Appl Basic Med Res 2014;4:3-6. https://doi.org/10.4103/ 2229-516X.125666

11. Wadhwa S, T Sanyal, Jain S, Nag T. Prenatal exposure to patterned and unpatterned noise differentially influences spatial behaviour, morphological and molecular changes in the chick brain. Int J Dev Neurosci 2012;30:663. https://doi. org/10.1016/j.ijdevneu.2012.03.305

12. Donofre AC, Silva IJO, Ferreira IEP. Sound exposure and its beneficial effects on embryonic growth and hatching of broiler chicks. Br Poult Sci 2020;61:79-85. https://doi.org/ 10.1080/00071668.2019.1673315

13. Chaudhury S, Nag TC, Wadhwa S. Effect of prenatal auditory stimulation on numerical synaptic density and mean synaptic height in the post hatch Day 1 chick hippocampus. Synapse 2009;63:152-9. https://doi.org/10.1002/syn.20585

14. Campbell DLM, De Haas EN, Lee C. A review of environmental enrichment for laying hens during rearing in relation to their behavioral and physiological development. Poult Sci 2019;98:9-28. https://doi.org/10.3382/ps/pey319

15. Chiandetti C, Vallortigara G. Chicks like consonant music. Psychol Sci 2011;22:1270-3. https://doi.org/10.1177/09567 97611418244

16. Crowe SF, Hale MW. Carryover effects associated with the single-trial passive avoidance learning task in the young chick. Neurobiol Learning Mem 2002;78:321-31. https://doi.org/10. 1006/nlme.2002.4069

17. Ying W, Yang Y, Han N, Xingu H. Implication of protein kinase $\mathrm{C}$ of the left intermediate medial mesopallium in memory impairments induced by early prenatal morphine exposure in one-day old chicks. Eur J Pharmacol 2017;795: 94-100. https://doi.org/10.1016/j.ejphar.2016.12.011

18. Abe H, Nagao K, Nakamura A, Inoue-Murayama M. Differences in responses to repeated fear-relevant stimuli between 
Nagoya and White Leghorn chicks. Behav Processes 2013; 99:95-9. https://doi.org/10.1016/j.beproc.2013.07.004

19. Gao H, Lu Y. Early development of intrinsic and synaptic properties of chicken nucleus laminaris neurons. Neuroscience 2008;153:131-43. https://doi.org/10.1016/j.neuroscience. 2008.01.059

20. Tong Q, McGonnell IM, Romanini CEB, et al. Effect of species-specific sound stimulation on the development and hatching of broiler chicks. Br Poult Sci 2015;56:143-8. https:// doi.org/10.1080/00071668.2014.1000822

21. Kathpalia P, Nag TC, Chattopadhyay P, et al. In ovo sound stimulation mediated regulation of BDNF in the auditory cortex and hippocampus of neonatal chicks. Neuroscience 2019;408:293-307. https://doi.org/10.1016/j.neuroscience. 2019.04.014

22. Forkman B, Boissy A, Meunier-Salaun MC, Canali E, Jones RB. A critical review of fear tests used on cattle, pigs, sheep, poultry and horses. Physiol Behav 2007;92:340-74. https:// doi.org/10.1016/j.physbeh.2007.03.016

23. Schütz KE, Forkman BR, Jensen P. Domestication effects on foraging strategy, social behaviour and different fear responses: a comparison between the red junglefowl (Gallus gallus) and a modern layer strain. Appl Anim Behav Sci 2001;74:114. https://doi.org/10.1016/S0168-1591(01)00156-3

24. Janczak AM, Riber AB. Review of rearing-related factors affecting the welfare of laying hens. Poult Sci 2015;94:145469. https://doi.org/10.3382/ps/pev123

25. Ahmed AA, Ma W, Ni Y, Qin Z, Zhao R. Embryonic exposure to corticosterone modifies aggressive behavior through alterations of the hypothalamic pituitary adrenal axis and the serotonergic system in the chicken. Horm Behav 2014;65: 97-105. https://doi.org/10.1016/j.yhbeh.2013.12.002

26. Franciosini MP, Claudio C, Proietti PC, Omar T, Giampaolo A. Plasma corticosterone levels in laying hens from three different housing systems: preliminary results. Ital J Anim Sci 2005;4:276-8. https://doi.org/10.4081/ijas.2005.276

27. Phi Van VD, Krause ET, Phi-Van L. Modulation of fear and arousal behavior by serotonin transporter (5-HTT) genotypes in newly hatched chickens. Front Behav Neurosci 2018;12: 284. https://doi.org/10.3389/fnbeh.2018.00284

28. Kumar V, Nag TC, Sharma U, Jagannathan NR, Wadhwa S. Differential effects of prenatal chronic high-decibel noise and music exposure on the excitatory and inhibitory synaptic components of the auditory cortex analog in developing chicks (Gallus gallus domesticus). Neuroscience 2014;269: 302-17. https://doi.org/10.1016/j.neuroscience.2014.03.061

29. Snowdon CT, Teie D, Savage M. Cats prefer species-appropriate music. Appl Anim Behav Sci 2015;166:106-11. https:// doi.org/10.1016/j.applanim.2015.02.012

30. Anderson GM, Feibel FC, Cohen DJ. Determination of serotonin in whole blood, platelet-rich plasma, platelet-poor plasma and plasma ultrafiltrate. Life Sci 1987;40:1063-70. https://doi.org/10.1016/0024-3205(87)90568-6

31. Bolhuis JE, Ellen ED, van Reenen CG, et al. Effects of genetic group selection against mortality on behavior and peripheral serotonin in domestic laying hens with trimmed and intact beaks. Physiol Behav 2009;97:470-5. https://doi.org/10.1016/ j.physbeh.2009.03.021

32. Roy S, Nag TC, Upadhyay AD, Mathur R, Jain S. Prenatal music stimulation facilitates the postnatal functional development of the auditory as well as visual system in chicks (Gallus domesticus). J Biosci 2014;39:107-17. https://doi.org/10.1007/ s12038-013-9401-0

33. Hedlund L, Jensen P. Incubation and hatching conditions of laying hen chicks explain a large part of the stress effects from commercial large-scale hatcheries. Poult Sci 2021;100:1-8. https://doi.org/10.1016/j.psj.2020.10.015 